\title{
Methods of the Definition Analysis of Fine Details of Images
}

\author{
S.V. Sai \\ Pacific national university \\ Russia
}

\section{Introduction}

Definition is one of the most important parameters of the color image quality and is determined by the resolution of channel brightness and chromaticity. System resolution is traditionally determined by a number of the television lines, calculated on the maximal spatial frequency value at which threshold contrast of the reproduced image is provided. Traditional methods of definition analysis are developed for standard analog color TV systems. Specific kind of distortions in digital vision systems is associated with the restrictions imposed by a particular compression algorithm, used for handling static and dynamic images.

Such distortions may lead to an inconsistency between a subjective estimate of the decoded image quality and the program estimate based on the standard calculation methods.

Till now, the most reliable way of image quality estimation is the method of subjective estimation which allows estimating serviceability of a vision system on the basis of visual e perception of the decoded image. Procedures of subjective estimation demand great amount $\mathcal{E}$ of tests and a lot of time. In practice, this method is quite laborious and restricts making o. control, tuning and optimization of the codec parameters.

The most frequently used root-mean-square criterion (RMS) for the analysis of static image ㄷ quality does not always correspond to the subjective estimation of fine details definition, D since a human vision system processes an image on local characteristic features, rather than $\stackrel{\Phi}{.}$ averaging it elementwise. In particular, RMS criterion can give "good" quality estimations in vision systems even at disappearance of fine details in low contrast image after a digital compression.

A number of leading firms suggest hardware and software for the objective analysis of OJ dynamic image quality of MPEG standard (Glasman, 2004). For example Tektronix PQA 300 స్ analyzer; Snell \& Wilcox Mosalina software; Pixelmetrix DVStation device. Principles of $\widetilde{\widetilde{\pi}}$ image quality estimation in these devices are various.

of For example, PQA 300 analyzer measures image quality on algorithm of "Just Noticeable \& Difference - JND", developed by Sarnoff Corporation. PQA 300 analyzer carries out a series $\underset{4}{0}$ of measurements for each test sequence of images and forms common PQR estimation on c the basis of JND measurements which is close to subjective estimations.

ญे To make objective analysis of image quality Snell \& Wilcox firm offers a PAR method -

O Picture Appraisal Rating. PAR technology systems control artifacts created by compression 
under MPEG-2 standard. The Pixelmetrix analyzer estimates a series of images and determines definition and visibility errors of block structure and PSNR in brightness and chromaticity signals.

The review of objective methods of measurements shows that high contrast images are usually used in test tables, while distortions of fine details with low contrast, which are most common after a digital compression, are not taken into account.

Thus, nowadays there is no uniform and reliable technology of definition estimation of image fine details in digital vision systems.

In this chapter new methods of the definition analysis of image fine details are offered. Mathematical models and criteria of definition estimation in three-dimensional color space are given. The description of test tables for static and dynamic images is submitted. The influence of noise on the results of estimations is investigated. The investigation results and recommendations on high definition adjustment in vision systems using JPEG, JPEG-2000 and MPEG-4 algorithms are given.

\section{Image Definition Estimation Criteria in Three-Dimensional Color Space}

The main difficulty in the objective criterion development is in the fact that threshold vision contrast is represented as a function of many parameters (Pratt, 2001). In particular, while analyzing the determined image definition, threshold contrast of fine details distinctive with an eye is represented as a function of the following parameters:

$$
K_{t h}=F\left(\alpha, t, C_{o}, C_{b}, \bar{\sigma}\right)
$$

where $\alpha$ is the object angular size, $t$ is the object presentation time, $C_{o}$ is the object color coordinates; $C_{b}$ is the background color coordinates, $\bar{\sigma}$ is the root-mean-square value of noise.

Solving the task it was necessary first to find such metric space where single changes of signals would correspond to thresholds of visual recognition throughout the whole color space, both for static, and for dynamic fine details.

One of the most widespread ways of color difference estimation of large details of static images is transformation of RGB space in equal contrast space where the area of dispersion of color coordinates transforms from ellipsoid to sphere with the fixed radius for the whole color space (Krivosheev \& Kustarev, 1990).

In this case the threshold size is equal to minimum perceptible color difference (MPCD) and keeps constant value independently of the object color coordinates.

The color error in equal color space, for example, in ICI 1964 system (Wyszecki, 1975) is determined by the size of a radius - vector in coordinates system and is estimated by the number of MPCD

$$
\varepsilon=3 \sqrt{\left(W_{o}^{*}-\widetilde{W}_{o}^{*}\right)^{2}+\left(U_{o}^{*}-\widetilde{U}_{o}^{*}\right)^{2}+\left(V_{o}^{*}-\widetilde{V}_{o}^{*}\right)^{2}}
$$

where $W_{o}^{*}, U_{o}^{*}, V_{o}^{*}$ is the color coordinates of a large object in a test image and $\widetilde{W}_{o}^{*}, \widetilde{U}_{o}^{*}, \widetilde{V}_{o}^{*}$ is the color coordinates in a decoded image; $W^{*}=25 Y^{1 / 3}-17$ is the brightness index; $U^{*}=13 W^{*}\left(u-u_{o}\right)$ and $V^{*}=13 W^{*}\left(v-v_{o}\right)$ is the chromaticity indexes; $u$ and $v$ is the 
chromaticity coordinates in D. Mac-Adam diagram (Mac Adam, 1974); $u_{o}=0,201$ and $v_{o}=$ 0,307 is the chromaticity coordinates of basic white color.

When comparing color fields located in "window" on a neutral background one can notice, that color differences (1) are invisible at $\varepsilon \leq 2 \ldots 3$ (MPCD) for the whole color space which is explained by the properties of equal color spaces (Novakovsky, 1988).

Color difference thresholds will increase with the reduction of objects sizes and will depend on the observable color. That is explained by the properties of visual perception. That's why equal color spaces practically are not used for the analysis of color transfer distortions of fine details since the property of equal spaces is lost.

As a result of the researches, the author (Sai, 2002) offers and realizes a method of updating (normalization) of equal space systems which are aimed to be used both for the analysis of large details distortions and for estimation of transfer accuracy of fine color details. Equal color space normalization consists in the following.

Determine color difference between two details of the image in size of a radius - vector

$$
\Delta E=3 \sqrt{\left(W_{1}^{*}-W_{2}^{*}\right)^{2}+\left(V_{1}^{*}-V_{2}^{*}\right)^{2}+\left(U_{1}^{*}-U_{2}^{*}\right)^{2}},
$$

where $W_{1}^{*} U_{1}^{*} V_{1}^{*}$ is the color coordinates of the 1-st object; $W_{2}^{*} U_{2}^{*} V_{2}^{*}$ is the color coordinates of the 2-nd object.

As against (1), equation (2) determines color difference between objects of one image, instead of between objects of images "before" and "after" digital processing.

If one of the objects is background, color contrast "object - background" is determined as follows:

$$
\Delta E=3 \sqrt{\left(W_{o}^{*}-W_{b}^{*}\right)^{2}+\left(V_{o}^{*}-V_{b}^{*}\right)^{2}+\left(U_{o}^{*}-U_{b}^{*}\right)^{2}}
$$

or in difference coordinates:

$$
\Delta E=\sqrt{\left(\Delta W^{*}\right)^{2}+\left(\Delta U^{*}\right)^{2}+\left(\Delta V^{*}\right)^{2}},
$$

where $\Delta W^{*}=3\left(W_{o}^{*}-W_{b}^{*}\right), \Delta U^{*}=3\left(U_{o}^{*}-U_{b}^{*}\right), \Delta V^{*}=3\left(V_{o}^{*}-V_{b}^{*}\right)$ is the difference values (MPCD) according to brightness and chromaticity indexes; $W_{o}^{*} U_{o}^{*} V_{o}^{*}$ is the object color coordinates; $W_{b}^{*} U_{b}^{*} V_{b}^{*}$ is the background color coordinates.

Assume, that the large detail of the image is recognized with an eye under the following condition:

$$
\Delta E \geq \Delta E_{t h}
$$

where $\Delta E_{t h}=2 \ldots 3(\mathrm{MPCD})$ is the threshold contrast which keeps constant value within the limits of the whole color space.

Further, we shall substitute (4) in (5) and convert to the following:

$$
\sqrt{\left(\frac{\Delta W^{*}}{\Delta E_{t h}}\right)^{2}+\left(\frac{\Delta U^{*}}{\Delta E_{t h}}\right)^{2}+\left(\frac{\Delta V^{*}}{\Delta E_{t h}}\right)^{2}} \geq 1
$$


The contrast sensitivity of human vision is reduced with the reduction of details sizes and threshold value $\left(\Delta E_{t h}\right)$ becomes dependent on the object size $\left({ }^{\alpha}\right)$, both in brightness, and chromaticity. Thus the criterion of fine details difference is defined as

$$
\sqrt{\left(\frac{\Delta W^{*}}{\Delta W_{t h}^{*}(\alpha)}\right)^{2}+\left(\frac{\Delta U^{*}}{\Delta U_{t h}^{*}(\alpha)}\right)^{2}+\left(\frac{\Delta V^{*}}{\Delta V_{t h}^{*}(\alpha)}\right)^{2}} \geq 1
$$

where $\Delta W_{t h}^{*}, \Delta U_{t h}^{*}$ and $\Delta V_{t h}^{*}$ is the threshold values according to brightness and chromaticity indexes which usually depend on color background coordinates, time of object presentation and noise level.

Write (7) in the following way:

$$
\sqrt{\left(\Delta \bar{W}^{*}\right)^{2}+\left(\Delta \bar{U}^{*}\right)^{2}+\left(\Delta \bar{V}^{*}\right)^{2}} \geq 1
$$

where $\Delta \bar{W}^{*}=\Delta W^{*} / \Delta W_{t h}^{*}, \Delta \bar{U}^{*}=\Delta U^{*} / \Delta U_{\text {th }}^{*}$ and $\Delta \bar{V}^{*}=\Delta V^{*} / \Delta V_{t h}^{*}$ is the normalized values of object - background contrast. Provided condition (8) is true, color difference between object and background is visible with an eye, hence fine details are perceptible.

Thus, transition from equal space into normalized equal space allows on the basis of criterion (8) to estimate objectively color difference of both large and fine details under preset conditions of color image supervision.

In vision systems where the receiver of the decoded images is the automatic device, and vision properties are not taken into account, the criterion of fine details difference can be received directly in three-dimensional space of $R G B$ signals:

$$
\sqrt{(\Delta R)^{2}+(\Delta G)^{2}+(\Delta B)^{2}} \geq \Delta K_{t h}
$$

where $\Delta K_{t h}$ is the threshold contrast value, which depends on device sensitivity and noise level at an output of a system.

In order to use criterion (8) in practice it is necessary to determine numerical values of fine details threshold contrast at which they are visible with an eye, depending on the size of details for the set of supervision conditions.

To solve this task it was required:

1. To develop a synthesis algorithm of the test image consisting of small static and dynamic objects with regulated contrast in MPCD values. 2. To develop a procedure of the experiment and on the basis of subjective estimations to determine threshold values of fine details contrast.

\section{Test Image Synthesis}

The author has developed a test image algorithm synthesis in equal color space, that allows to set initial contrast of object - background directly in color thresholds, that is basically different from the known ways of synthesis when the image contrast is set by the percentage of object brightness to background brightness.

The synthesis algorithm consists in the following. 
At the first stage form, sizes, spatial position and color coordinates $\left(W^{*} U^{*} V^{*}\right)$ of objects and background for the basic first frame of test sequence are set. The vectors of movement are set for the subsequent frames.

At the second stage the transformation $\left\{W_{m, i, j}^{*} U_{m, i, j}^{*} V_{m, i, j}^{*}\right\} \rightarrow\left\{R_{m, i, j} G_{m, i, j} B_{m, i, j}\right\}$ which is necessary for visualization of the initial sequence on the screen and for submission of digital $R G B$ signals on the input of the system under research is carried out for each frame of test sequence on the basis of mathematical model which have been developed. Where $m$ is the frame number; $i$ and $j$ is the pixels numbers in columns and lines of image.

At the third stage, cyclic regeneration of the $M$ frames with the set frequency $\left(f_{\text {frame }}\right)$ is carried out. When reproducing the test sequence, dynamic objects move on the set trajectory to the number of pixels having been determined by the motion vector.

On the basis of the above described algorithm the test table and video sequences are developed into which all the necessary elements for the quality analysis of fine details of static and dynamic images are included.

Let's consider the basic characteristics of the test table which is developed for the quality analysis of static images.

The table represents the image of CIF format (360×288), which is broken into 6 identical fragments $(120 \times 144)$. Each fragment of the table contains the following objects: a) horizontal, vertical and inclined lines with the stripes width of 1, 2, 3 or more 3 pixels; b) single small details of rectangular form. Objects of the image are located on a grey unpainted background.

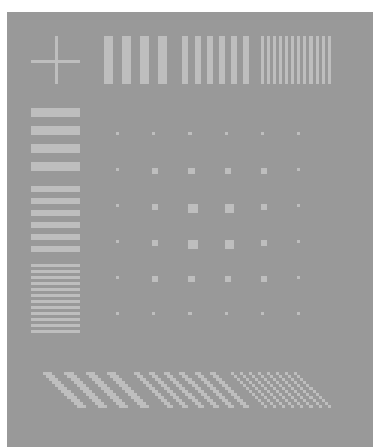

a)

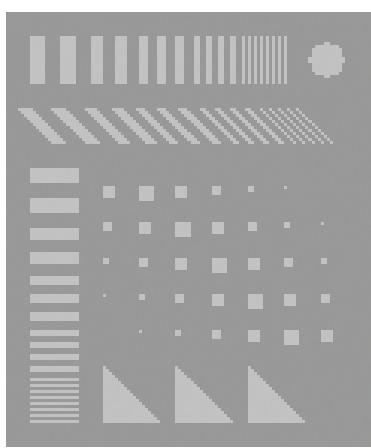

b)

Figure 1. A fragments of the test image: a) 1-st variant; a) 2-nd variant

The object - background brightness $\Delta W^{*}$ contrast is set by MPCD number for the 1-st and the 2-nd fragments

$$
\Delta W^{*}= \pm 3\left(W_{o}^{*}-W_{b}^{*}\right), \text { at } \Delta U^{*}=0 \text { and } \Delta V^{*}=0 .
$$

The object - background chromaticity $\Delta U^{*}$ contrast is set by MPCD number for the 3-rd and the 4-th fragments

$$
\Delta U^{*}= \pm 3\left(U_{o}^{*}-U_{b}^{*}\right) \text {, at } \Delta W^{*}=0 \text { and } \Delta V^{*}=0 .
$$

The object - background chromaticity $\Delta V^{*}$ contrast is set by MPCD number for the 5-th and the 6-th fragments 


$$
\Delta V^{*}= \pm 3\left(V_{o}^{*}-V_{b}^{*}\right), \text { at } \Delta W^{*}=0 \text { and } \Delta U^{*}=0 .
$$

As an example, fragments $(120 \times 144)$ of the test image on brightness for two variants of tables are shown on Figure 1.

Three types of test video sequences with formats $360 \times 288,720 \times 576$ and $1440 \times 1152$ pixels are developed for the quality analysis of dynamic images.

The table with a format $360 \times 288$ is used as the basis (I-frame) of test video sequence. The sequence consists of 12 frames cyclically repeated at a certain frequency $f_{\text {frame }}=30 \mathrm{~Hz}$.

Spatial coordinates of the $m$ - frame objects are displaced relatively the frame number $m-1$ on the value of motion vector. During the sequence regeneration all the details of the image of the test table become dynamic.

In test sequence with a format $720 \times 576$ every frame consists of 4 fragments of a format $360 \times 288$. And, at last, for sequence of a format $1440 \times 1152$ every frame contains 4 fragments of a format $720 \times 576$.

\section{Experimental Estimation of Visual Thresholds}

The test table and sequence with format $352 \times 288$ are synthesized to determine the threshold of visual perception of the image fine details.

The developed user program interface allows adjusting the following image parameters: background brightness, object contrast on brightness and chromaticity indexes.

Threshold values of contrast for static details on brightness and chromaticity indexes were received experimentally with the help of subjective estimations with the following technique.

1. The test image with adjustable values of color contrast on axis $\Delta W^{*}$ with step 1 MPCD and on axes $\Delta U^{*}$ and $\Delta V^{*} U$ with step 2 MPCD was offered to the observer.

2. During the experiment the observer changed the contrast value beginning with the minimal until the stripes became distinct.

3. As an estimation criterion of threshold contrast the following condition was set: the stripes should be distinguishable with an eye in comparison with the previous image i.e. at which contrast was one step lower.

4. Under condition (3) the observer fixed value of contrast at which, in his opinion, sufficient "perceptibility" of lines was provided.

Students and employees of Khabarovsk state technical university (Pacific National University) participated in the experiments.

\begin{tabular}{|c|c|c|c|c|}
\hline$\delta$ & $>3$ & 3 & 2 & 1 \\
\hline$\Delta W_{t h}^{*}$ & 2 & 3 & 4 & 6 \\
\hline$\Delta U_{t h}^{*}$ & 26 & 34 & 48 & 72 \\
\hline$\Delta V_{t h}^{*}$ & 24 & 36 & 52 & 76 \\
\hline
\end{tabular}

Table 1. Dependences of threshold contrast from the size of objects 
Table 1. shows subjective average estimations of threshold contrast from the size $(\delta)$ of objects for background brightness is $W_{b}^{*}=80 \mathrm{MPCD}$, arithmetic-mean value being received by estimation results of 20 observers.

In the table the size of objects is set by pixels number, and the threshold value by the MPCD number. For example, at the minimal sizes of lines $(\delta=1)$ the average value of a visual threshold on brightness index is equal to $6 \mathrm{MPCD}$ and on chromaticity index it is equal to 72 and 76 MPCD.

For example, at the minimal sizes of stripes the average value of a visual threshold on brightness index is equal to $6 \mathrm{MPCD}$ and on chromaticity index it is equal to 72 and 76 MPCD.

The results of the experiments show, that values of threshold contrast on an unpainted background on axes $\Delta U^{*}$ and $\Delta V^{*}$ are approximately identical, and exceed values of thresholds on axis $\Delta W^{*}$ in $10 \ldots 13$ times. Change of background brightness from 70 up to 90 MPCD does not essentially influence the thresholds of fine details visual perception.

Experimental estimations of color thresholds in $L^{*} u^{*} v^{*}$ system show, that estimations on coordinates of chromaticity $u^{*}$ and $v^{*} 1.5 \ldots 1.8$ times differ. Therefore the use of $W^{*} U^{*} V^{*}$ system is more preferable.

The values of threshold contrast for mobile details of test sequence are received by experimentally with the help of subjective estimations by the following technique.

During the experiment the observer changed of contrast value, beginning with the minimal until the mobile objects became distinct.

The results of the experiments show that, at movement of objects, contrast threshold values in comparison with the data of Table 1, increase, depending on $t$ according to function $f(t)=1 /\left(1-e^{-t / \vartheta}\right)$, where $\vartheta=0,05$ is the time of vision inertia; $t$ is the time interval, during which the object moves on a certain number of pixels set by the vector.

In particular, at $t=0,033$ ( $f_{\text {frame }}=30 \mathrm{~Hz}$ ) values of contrast threshold of fine details have increased approximately in $1,8 \ldots 2$ times.

Thus, the received experimental data allow using criterion (8) in practice as an objective estimation of transfer accuracy of both static and dynamic fine details of the test image.

\section{Analysis of Definition and Distortions of Test Table Fine Details}

The analysis of definition and distortions of test table fine details consists of the following stages.

At the first stage, the test sequence of 12 image frames in $R G B$ signal space, where $W^{*} U^{*} V^{*}$ space is used as initial object color coordinates, is synthesized.

Contrast of stripes image and fine details two - three times exceeds the threshold values. Such choice of contrast is caused by the fact that in the majority of cases fine details with low contrast are more distorted during digital coding and images transfer.

At the second stage, digital RGB signals of test sequence move on an input of the test system and are processed using coding algorithm. 
At the third stage after decoding, the test sequence is restored and $\widetilde{R}_{m, i, j}, \widetilde{G}_{m, i, j}, \widetilde{B}_{m, i, j}$ signals are transformed into $\widetilde{W}_{m, i, j}^{*}, \widetilde{U}_{m, i, j}^{*}, \widetilde{V}_{m, i, j}^{*}$ signals for each frame. All 12 frames of the restored sequence write in a RAM of the analyzer.

At the fourth stage, contrast and distortions of fine details are measured by the local fragments of the restored image, and definition estimation is obtained by the objective criteria.

Let's consider a measurement method of stripes contrast of the first image frame.

For an estimation of definition impairment it is necessary to measure contrast for each fragment of the decoded image of stripes with the fixed size and to compare the received value to threshold value. We assume that stripes are distinguished by the observer, if the condition is satisfied:

$$
\Delta \overline{\widetilde{E}}(\delta, k)=\sqrt{\left(\frac{\Delta \overline{\widetilde{W}}^{*}(\delta, k)}{\Delta W_{t h}^{*}(\delta)}\right)^{2}+\left(\frac{\Delta \overline{\widetilde{U}}^{*}(\delta, k)}{\Delta U_{t h}^{*}(\delta)}\right)^{2}+\left(\frac{\Delta \overline{\widetilde{V}}^{*}(\delta, k)}{\Delta V_{t h}^{*}(\delta)}\right)^{2}} \geq 1
$$

where $\Delta \overline{\widetilde{E}}(\delta, k)$ is the average normalized value of stripes contrast, average on the $k$ "window" area of the image; $\Delta \overline{\widetilde{W}}^{*}, \Delta \overline{\widetilde{U}}^{*}$ and $\Delta \overline{\widetilde{V}}^{*}$ is the average values of contrast on brightness and chromaticity indexes; $k$ - the parameter determining the type the "window" under analysis ( $k=0$ - vertical stripes, $k=1$ - horizontal, $k=2$ - sloping); $\Delta W_{t h}^{*}(\delta), \Delta U_{t h}^{*}(\delta)$ and $\Delta V_{t h}^{*}(\delta)$ is the contrast threshold values from Table 1 .

Since the test image is divided into fragments on brightness and chromaticity indexes, the criteria of distinction of stripes on each coordinate are determined as follows:

$$
\Delta \overline{\widetilde{E}}_{W^{*}}(\delta)=\frac{\Delta \overline{\widetilde{W}}^{*}(\delta)}{\Delta W_{t h}^{*}(\delta)} \geq 1, \Delta \overline{\widetilde{E}}_{U^{*}}(\delta)=\frac{\Delta \overline{\widetilde{U}}^{*}(\delta)}{\Delta U_{t h}^{*}(\delta)} \geq 1, \Delta \overline{\widetilde{E}}_{V^{*}}(\delta)=\frac{\Delta \overline{\widetilde{V}}^{*}(\delta)}{\Delta V_{t h}^{*}(\delta)} \geq 1,
$$

where making calculations the minimal value of contrast from the three $(k)$ "windows" under analysis is chosen on each color coordinate, which allows taking into account the influence of spatial orientation of lines for decoding accuracy.

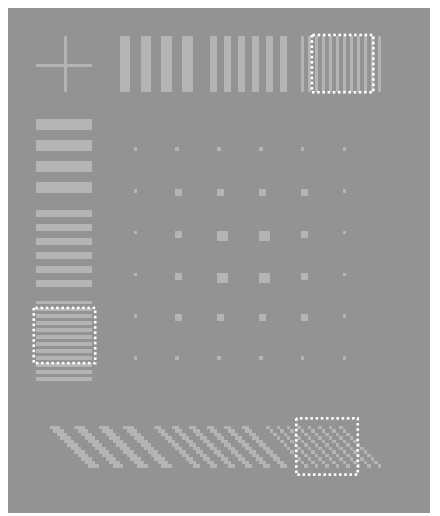

Figure 2. Image fragment "windows" under analysis 
Figure 2 shows the example of spatial position of the image fragment "windows" under analysis on the brightness index with contrast $\Delta W_{t h}^{*}=18$ MPCD which is three times higher than the threshold value for the finest details $(\delta=1)$.

Average contrast values on brightness and chromaticity indexes are equal to the initial values if there are no distortions. In this case, contrast of all the "windows" of the test image under analysis three times exceeds threshold values and, hence, definition does not become worse.

Average contrast value of the "windows" of the test image under analysis decreases, if there are distortions. But, if the contrast on brightness or chromaticity index becomes less than threshold value, i.e. conditions (10) are not satisfied, the conclusion is made that the observer does not distinguish fine details.

Finally, minimal size of stripes with the contrast which satisfies criteria (10) makes it possible to determine maximum number of distinct elements of the image that constitutes the image definition estimation on brightness and chromaticity.

It is obvious, that the estimation by criteria (10) depends on the initial image contrast. In particular the stripes contrast decrease on $1 \ldots 2$ thresholds gives "bad" results when using test image with low contrast. But when the initial contrast exceeds threshold values 10 times, definition impairment is not observed in such contrast decrease.

Thus, the criterion (8) gives an objective estimation of definition impairment of fine details of low contrast image.

To exclude initial contrast influence on indeterminacy of estimations, we should take the following equation for brightness index:

$$
\varepsilon_{W^{*}}(\delta)=\frac{1}{\Delta W_{t h}^{*}(\delta) \cdot N} \sum_{i=0}^{N-1}\left|\Delta W^{*}(\delta)-\Delta \widetilde{W}_{i}^{*}(\delta)\right|<Q_{W^{*}}
$$

where $\varepsilon_{W^{*}}(\delta)$ is the threshold- normalized deviation of contrast from the initial value; $Q_{W^{*}}$ is the quality parameter determining admissible values of contrast decrease on brightness index; $N$ is the pixels number in the "window" under analysis.

Calculations on chromaticity are made on analogy.

Calculations having been made, the program analyzer compares the results received with the quality rating in a ten-point scale and establishes estimation.

It is shown in (Sai, 2003) that high-quality reproduction of fine details with the rating not less than $6 \ldots 7$ points, is obtained under the following conditions: a) contrast reduction of stripes on brightness should be not more than $50 \%$ of the threshold values for the stripes width of 1 pixel or more; b) contrast reduction of stripes on chromaticity should be not more than $75 \%$ of the threshold values for the stripes width of 3 pixels or more, i.e.

$$
\varepsilon_{W^{*}}(\delta \geq 1)<0,5 ; \varepsilon_{U^{*}, V^{*}}(\delta \geq 3)<0,75
$$

The experimental results of the images quality analysis in different compression systems show that, when these criteria are met, the reduction of the visual sharpness of fine details is only barely visible or almost imperceptible.

The developed method differs from the known in the fact that contrast of fine details at the exit of a system is estimated by the threshold- normalized average value of the "window" area of the stripes image under analysis, but not by the amplitude value of the first harmonic of brightness and chromaticity signals. 
Object - background initial contrast is also set not by the maximal value, but in two - three times exceeding threshold value that allows to estimate the effectiveness of coding system in up to threshold area where distortions are the most essential.

Thus, the offered method allows estimating objectively the reduction of the visual sharpness since it takes into account thresholds of visual perception of fine details and possible fluctuations of color coordinates caused by linear distortions of signals and noise presence in digital system.

In image coding digital systems using nonlinear transformations not only linear reduction of high-frequency component of decoded $R G B$ signals is possible, but also nonlinear distortions may occur.

Therefore, in some cases, the estimation of contrast reduction by criteria (12) can lead to incorrect results.

To take into account the influence of nonlinear distortions on objectivity of estimations the following decision is offered.

In addition to estimations (12), the normalized average deviation of reproduced color coordinates relative to the initial ones in the image "window", for example, on brightness is offered to estimate:

$$
\Delta_{W^{*}}(\delta)=\frac{1}{\Delta W_{t h}^{*}(\delta) \cdot N} \sum_{i=0}^{N-1}\left|\widehat{W}_{i}^{*}(\delta)-W_{i}^{*}(\delta)\right|
$$

It is shown in (Sai, 2003) that in order to provide high-quality reproduction of fine details with the rating not less than $6 \ldots 7$ points, it is necessary to satisfy the following conditions in addition to criteria (12): a) the root-mean-square deviation of brightness coordinates in all "windows" under analysis must be not more than $30 \%$; b) the root-mean-square deviation of chromaticity coordinates not more than $50 \%$ for the details not less than three pixels in size.

$$
\Delta_{W^{*}}(\delta \geq 1)<0,3 ; \quad \Delta_{U^{*}, V^{*}}(\delta \geq 3)<0,5
$$

Consider the method of distortions estimation of fine single details of a rectangular form. For the test image fragment, for example on brightness, find the normalized average deviation of object contrast and initial value on the object area:

$$
\eta_{W^{*}}(\delta)=\frac{1}{\Delta W_{t h}^{*}(\delta) \cdot N} \sum_{i=0}^{N-1}\left|\Delta \widehat{W}_{i}^{*}(\delta)-\Delta W^{*}(\delta)\right|
$$

As against (11), number $N$ is determined by the image "window" with a single object being included into it. For example, at the analysis of distortions of point object the "window" size is $1 \times 1$ pixels. At the analysis of distortions of object $2 \times 2$ pixels in size, the "window" size is $2 \times 2$, etc.

It is obvious from the experiments, that in order to ensure high-quality reproduction of fine details with the rating not less than $6 \ldots 7$ points, it is necessary to satisfy the following conditions: a) the root-mean-square deviation on brightness must be not more than 1,5 for all the details; b) the root-mean-square deviation on chromaticity must be not more than 0,8 for the details 3 or more pixels in size.

$$
\eta_{W^{*}}(\delta \geq 1)<1,0 ; \quad \eta_{U^{*}, V^{*}}(\delta \geq 3)<0,5
$$


Thus a program analyzer can estimate visual quality of reproduction of striped lines and fine details of the test image by criteria (12), (14) and (16).

Table 1 shows the experimental dependence of parameters (11), (13) and (15) from quality rating.

Results are received after JPEG compression of the image in Adobe Photoshop 5 using tenpoint scale of quality. The results are received for the test image with fine details contrast exceeding threshold values two times. Thus according to Table 1., it is possible to estimate the quality rating for each of the six parameters.

The average quality rating of each frame of the test sequence is calculated as follows:

$$
Q_{m}=\frac{1}{6} \sum_{i=1}^{6} Q_{i}
$$

\begin{tabular}{|c|c|c|c|c|c|c|c|c|c|c|c|}
\hline \multirow{2}{*}{$\mathrm{Q}$} & 1 & 2 & 3 & 4 & 5 & 6 & 7 & 8 & 9 & 10 & \\
\hline & \multicolumn{2}{|l|}{ Low } & \multicolumn{3}{|c|}{ Medium } & \multicolumn{2}{|l|}{ High } & \multicolumn{3}{|c|}{ Maximum } & \multirow{4}{*}{$\delta=1$} \\
\hline$\varepsilon_{W^{*}}$ & 1,006 & 0,966 & 0,948 & 0,690 & 0,498 & 0,225 & 0,099 & 0,071 & 0,012 & 0,013 & \\
\hline$\Delta_{W^{*}}$ & 0,690 & 0,700 & 0,686 & 0,627 & 0,519 & 0,338 & 0,240 & 0,145 & 0,083 & 0,015 & \\
\hline$\eta_{W}{ }^{*}$ & 2,039 & 2,039 & 1,722 & 1,617 & 1,512 & 1,409 & 0,998 & 0,295 & 0,097 & 0,001 & \\
\hline$\varepsilon_{U^{*}}$ & 1,528 & 1,617 & 1,569 & 1,073 & 0,772 & 0,557 & 0,391 & 0,241 & 0,009 & 0,002 & \multirow{3}{*}{$\delta=3$} \\
\hline$\Delta_{U^{*}}$ & 0,960 & 0,955 & 0,917 & 0,688 & 0,505 & 0,432 & 0,331 & 0,238 & 0,143 & 0,053 & \\
\hline$\eta_{U^{*}}$ & 1,124 & 1,070 & 1,024 & 1,143 & 0,456 & 0,460 & 0,477 & 0,299 & 0,124 & 0,047 & \\
\hline
\end{tabular}

Table 1. The experimental dependence of parameters from quality rating

Consider a measurement technique for mobile objects of the test sequence.

For an estimation of definition it is necessary to calculate average values of contrast deviation of stripes on brightness and chromaticity for every $m$ of the frame of test sequence and to estimate average value for the set of 12 frames:

$$
\varepsilon_{W^{*}}(\delta)=\frac{1}{M} \sum_{m=1}^{M}\left[\frac{1}{N} \sum_{i=0}^{N-1} \frac{\left|\Delta W^{*}(\delta)-\Delta \widetilde{W}_{i}^{*}(\delta, m)\right|}{\Delta W_{t h}^{*}(\delta) \cdot f(t)}\right]
$$

where $M=12$ is the frames number; $f(t)$ is the function taking into account recession of contrast - sensitive vision characteristic depending on objects presentation time.

Reduction of stripes contrast on chromaticity is calculated similarly.

Calculations (17) having been made, conditions (12) are checked.

If (14) is satisfied on brightness and chromaticity, the decision is made, that the observer distinguishes fine mobile details and definition reduction is slightly visible.

For the estimation of parameters (13) and (15) average values on 12 frames of test sequence are calculated on analogy to the equation (17). 


\section{Noise Influence Analysis}

The developed criteria of image quality estimation are received without taking into account noise in $R G B$ signals. Hence the correctness of the results is true in the case when noise level in the received image is small enough.

The analysis of noise influence in a digital video system can be divided into two parts: analysis in up to threshold area and analysis in higher of threshold area.

In the up to threshold area the transfer quality of coded video data is high, and noise presence in the system results only in small fluctuations of $R G B$ signals.

But, if the noise level and probability of mistakes exceed the threshold value, abrupt image quality impairment is observed because of possible changes of pixels spatial position and distortions of signal peak values.

In order to analysis noise influence on the image definition reduction in the up to threshold area take advantage of the following assumptions:

1. Interaction of signals and noise is additive.

2. Density distribution law of stationary noise probabilities is close to the normal law.

3. Noise in $R G B$ signals of the decoded image is not correlative.

Noise in the system results in "diffusion" of both objects color coordinates and background in the decoded image. Thus a point in RGB space is transformed into ellipsoid with semi axis. Their values are proportional to root-mean-square noise levels.

Calculating the stripes contrast, make the following transformation:

$$
\left\{R_{m, i, j} G_{m, i, j} B_{m, i, j}\right\} \rightarrow\left\{W_{m, i, j}^{*} U_{m, i, j}^{*} V_{m, i, j}^{*}\right\} .
$$

Hence, values of equal coordinates become random variables with root-mean-square deviations: $\sigma_{W^{*}}, \sigma_{U^{*}}, \sigma_{V^{*}}$.

Dispersions of $W^{*}, U^{*}$ and $V^{*}$ coordinates are received with the help of a linearization method (Ventzel \& Ovtharov, 2000) of the functions $W^{*}=25 Y^{1 / 3}-17, U^{*}=13 W^{*}\left(u-u_{0}\right)$ and $V^{*}=13 W^{*}\left(v-v_{0}\right)$.

Define dispersion of brightness index $W^{*}=25 Y^{1 / 3}-17$. Linearization of the functions $W^{*}=\varphi\left(Y^{1 / 3}\right)$ is the approached representation of this function by first two members of Taylor series. In this case, the dispersion $\sigma_{W^{*}}^{2}$ can be found in the approximate way:

$$
\sigma_{W^{*}}^{2} \approx\left(\frac{\partial W^{*}}{\partial Y}\right)^{2} \cdot \sigma_{Y}^{2}=\left(\frac{25}{3 \cdot Y^{2 / 3}}\right)^{2} \cdot \sigma_{Y}^{2},
$$

where, the brightness coordinate is determined by linear transformation: $Y=0,299 L_{R}+0,587 L_{G}+0,114 L_{B}$.

Therefore

$$
\sigma_{Y}^{2}=0,299^{2} \cdot \sigma_{R}^{2}+0,587^{2} \cdot \sigma_{G}^{2}+0,114^{2} \cdot \sigma_{B}^{2}
$$


Define dispersion of chromaticity index $U^{*}$ with the help of a linearization method of the function $U^{*}=13 W^{*}\left(u-u_{0}\right)$

$$
\sigma_{U^{*}}^{2} \approx\left(\frac{\partial U^{*}}{\partial R}\right)^{2} \cdot \sigma_{R}^{2}+\left(\frac{\partial U^{*}}{\partial G}\right)^{2} \cdot \sigma_{G}^{2}+\left(\frac{\partial U^{*}}{\partial B}\right)^{2} \cdot \sigma_{B}^{2},
$$

where derivatives are found in the following way:

$$
\begin{aligned}
& \frac{d U^{*}}{d R}=13\left(\frac{25 a_{4}}{3 Y^{2 / 3}}\left(u-u_{0}\right)+\frac{a_{1} T-b_{1} U}{T^{2}} W^{*}\right) ; \\
& \frac{d U^{*}}{d G}=13\left(\frac{25 a_{5}}{3 Y^{2 / 3}}\left(u-u_{0}\right)+\frac{a_{2} T-b_{2} U}{T^{2}} W^{*}\right) ; \\
& \frac{d U^{*}}{d B}=13\left(\frac{25 a_{6}}{3 Y^{2 / 3}}\left(u-u_{0}\right)+\frac{a_{3} T-b_{3} U}{T^{2}} W^{*}\right),
\end{aligned}
$$

where $T=U+V+W ; u=U / T ; v=V / T$;

$U=a_{1} R+a_{2} G+a_{3} B ; V=a_{4} R+a_{5} G+a_{6} B ; W=a_{7} R+a_{8} G+a_{9} B ;$

$a_{1} \ldots a_{9}$ is the constants, and $b_{1}=a_{1}+a_{4}+a_{7} ; b_{2}=a_{2}+a_{5}+a_{8} ; b_{3}=a_{3}+a_{6}+a_{9}$.

Dispersion of chromaticity index $V^{*}$ is calculated similarly.

Estimate of noise influence on visual sharpness reduction of the test image (Figure 1).

As, the test image is divided into fragments on indexes of brightness and chromaticity, rootmean-square deviations of difference color coordinate for each fragment can be estimated of the following expressions:

$$
\begin{aligned}
& \sigma_{\Delta W^{*}} \approx 3 \sqrt{\sigma_{W_{o}^{*}}^{2}+\sigma_{W_{b}^{*}}^{2}}, \text { at } \Delta U^{*}=0 \text { and } \Delta V^{*}=0 ; \\
& \sigma_{\Delta U^{*}} \approx 3 \sqrt{\sigma_{U_{o}^{*}}^{2}+\sigma_{U_{b}^{*}}^{2}}, \text { at } \Delta W^{*}=0 \text { and } \Delta V^{*}=0 ; \\
& \sigma_{\Delta V^{*}} \approx 3 \sqrt[3]{\sigma_{V_{o}^{*}}^{2}+\sigma_{V_{b}^{*}}^{2}} \text {, at } \Delta W^{*}=0 \text { and } \Delta U^{*}=0 .
\end{aligned}
$$

Define criterion at which the observer distinguishes image stripes with noise.

The known «three sigma » rule is used to solve the task. This rule means that deviation probability of a random variable $X$ from its mean value not less than three sigma, provided the law of distribution is close to normal, does not exceed 1/9.

Criteria, at which the observer distinguishes stripes in the test image with noise on brightness, are found in the following way:

$$
\frac{\left|\Delta W^{*}(\delta)\right|-3 \sigma_{\Delta W^{*}} \cdot \varphi_{W^{*}}(\delta)}{\Delta W_{t h}^{*}(\delta)} \geq 1
$$

where $\varphi(\delta)$ is the weight function.

Criteria of chromaticity indexes $U^{*}$ and $V^{*}$ is calculated similarly.

Introduction of weight function into (18) is caused by the fact that vision contrast sensitivity decreases with the reduction of the details sizes and hence the influence of noise on their perceptibility is greater. 
Experimental research results have shown that the maximal value of weight function $(\varphi(\delta)=1)$ corresponds to the minimal size $(\delta=1)$ of stripes, and weight function values decrease with the increase of the stripes size. This is proportional to the reduction of threshold values (Table 1).

The numerical solution of the developed mathematical model allows estimating the influence of additive noise on definition reduction depending on root-mean-square values of noise in RGB signals on the system output.

Dependences of root-mean-square deviations of color coordinates on brightness and chromaticity from $\left(\sigma \approx \sigma_{R} \approx \sigma_{G} \approx \sigma_{B}\right)$ are shown in Table 2, provided that noise levels in $R$, $G$ and $B$ signals are approximately identical.

Value $(\sigma)$ is given in percentage ratio to maximal amplitude of $R, G$ and $B$ signals.

Objects color coordinates of the test image with the contrast equal to threshold value for the details with the minimal sizes are used in calculations, i.e., $W_{b}^{*}=80 \mathrm{MPCD}, \Delta W^{*}=6 \mathrm{MPCD}$, $\Delta U^{*}=72$ MPCD and $\Delta V^{*}=76$ MPCD.

\begin{tabular}{|c|c|c|c|c|c|c|c|c|c|c|}
\hline$\sigma_{\%}$ & 0.2 & 0.4 & 0.6 & 0.8 & 1.0 & 1.2 & 1.4 & 1.6 & 1.8 & 2.0 \\
\hline$\sigma_{W^{*}}$ & 0.31 & 0.61 & 0.92 & 1.22 & 1.53 & 1.84 & 2.14 & 2.45 & 2.76 & 3.06 \\
\hline$\sigma_{U^{*}}$ & 1.20 & 2.40 & 3.60 & 4.81 & 6.01 & 7.19 & 8.43 & 9.61 & 10.8 & 12.1 \\
\hline$\sigma_{V^{*}}$ & 2.10 & 4.20 & 6.30 & 8.41 & 10.5 & 12.6 & 14.8 & 16.8 & 18.9 & 21.0 \\
\hline
\end{tabular}

Table 2. Dependences of root-mean-square deviations of $W^{*}, U^{*}$ and $V^{*}$ color coordinates

The results received allow to estimate the influence of noise in $R G B$ signals on system output on threshold contrast increase (18) and, hence, on impairment of visual sharpness.

For example, to make finest details of the image distinguished by the observer at a relative noise level in $R G B$ signals is $\sigma_{R} \approx \sigma_{G} \approx \sigma_{B}=\sigma=2 \%(\Psi=34 \mathrm{~dB})$ their contrast should be increased on 9 MPCD in brightness and on 36 MPCD and 63 MPCD in chromaticity.

Selective average dispersion values on indexes of brightness and chromaticity are used for the proof of a correctness of the developed mathematical model.

For example, for an brightness index

$$
\bar{\sigma}_{W^{*}}^{2} \approx \frac{1}{N^{2}} \sum_{i=1}^{N} \sum_{j=1}^{N}\left(W_{i, j}^{*}-\bar{W}^{*}\right)^{2},
$$

where, $\bar{W}^{*}$ is the selective average value of $W^{*}$ coordinate in a window with size $N \times N$ pixels. Comparison calculated both experimental data proves a correctness of the developed mathematical model of noise transformation. Insignificant deviations into comparison results do not exceed $3 \ldots 5 \%$ and explained of a linearization method errors.

\section{Practical Results}

The developed methods are used in practice for the analysis and adjustment of video systems parameters, to get high quality transfer and reproduction of images fine details. The results of the analysis are given below and the recommendations on adjustment for high definition in vision systems using JPEG, JPEG-2000 and MPEG-4 algorithms are offered. 
The experimental analysis of coding quality of static images is carried out by the following technique.

At the first stage the influence of the coder parameters on the decoded image quality of the test table is analyzed with the help of a computer analyzer.

The computer analyzer calculated the following dependences of image quality parameters on the coder adjustment parameters: a) reduction of stripes contrast (11) on brightness and chromaticity; b) average deviation (13) of stripes color coordinates; c) average deviation (15) of a single object contrast.

At the second stage the coder parameter at which the results of the analysis correspond to high quality rating of $(Q \geq 6 \ldots 7)$ is selected.

At the third stage, the efficiency of digital compression of test images is estimated. Original test photo images containing $50 \ldots 70$ percent of thin structural elements were used at the experiment.

\section{Quality analysis of JPEG and JPEG2000 images}

The results of quality analysis of JPEG and JPEG2000 images coded in Adobe Photoshop CS are given below. In Table 3 one can see reproduction quality parameters of stripes and fine details of the test image on brightness for low, average and high rating quality.

In column (Var) values of adjustment parameters of images quality, being used in Adobe Photoshop CS are shown.

Fragments of the test image with various quality rating are shown on Figure 3.

\begin{tabular}{|c|c|c|c|c|c|c|c|c|c|}
\hline JPEG & $\varepsilon_{W^{*}}$ & $\Delta_{W^{*}}$ & $\eta_{W^{*}}$ & Var & JPEG2000 & $\varepsilon_{W^{*}}$ & $\Delta_{W^{*}}$ & $\eta_{W^{*}}$ & Var \\
\hline$Q=2$ & 0,97 & 0,70 & 2,04 & $\mathbf{4}$ & $Q=2$ & 1,09 & 0,69 & 1,72 & $\mathbf{2 5}$ \\
\hline$Q=4$ & 0,69 & 0,63 & 1,62 & $\mathbf{7}$ & $Q=4$ & 0,93 & 0,57 & 1,62 & $\mathbf{3 0}$ \\
\hline$Q=7$ & 0,10 & 0,24 & 0,99 & $\mathbf{9}$ & $Q=7$ & 0,19 & 0,13 & 0,31 & $\mathbf{6 5}$ \\
\hline
\end{tabular}

Table 3. Quality parameters JPEG and JPEG2000

The analysis of the results received shows that such adjustment parameters as: Var $\geq 9$ at JPEG compression and Var $\geq 65$ at JPEG2000 compression are to be established for providing high images definition in Adobe Photoshop CS.

The quality analysis of JPEG and JPEG2000 images coded in ACD See 8 is done in the similar way. The analysis of the results received shows that such adjustment parameters as: Var $\geq 80$ at JPEG compression and Var $=$ Compression ratio $\leq 30$ at JPEG2000 compression are to be established for providing high images definition in ACD See 8.

\begin{tabular}{|c|c|c|c|c|l|}
\hline \multirow{2}{*}{} & \multicolumn{2}{|c|}{ Adobe Photoshop CS } & \multicolumn{2}{|c|}{ ACD See 8 } & \\
\cline { 2 - 5 } & JPEG (9) & JPEG2000 (65) & JPEG (80) & JPEG2000 (30) & \\
\hline$C f$ & 6,4 & 3,1 & 12 & 27 & Lena \\
\hline$C f$ & 8,5 & 7,0 & 13 & 30 & Barbara \\
\hline
\end{tabular}

Table 4. Test images compression factors

As an example of compression efficiency of test images of Lena and Barbara at the established parameters of JPEG and JPEG2000 codecs on high definition are shown in table 4 , where $\mathrm{Cf}$ is the compression factor. The initial format of test images is equal $512 \times 512 \times 3$ Bytes. 

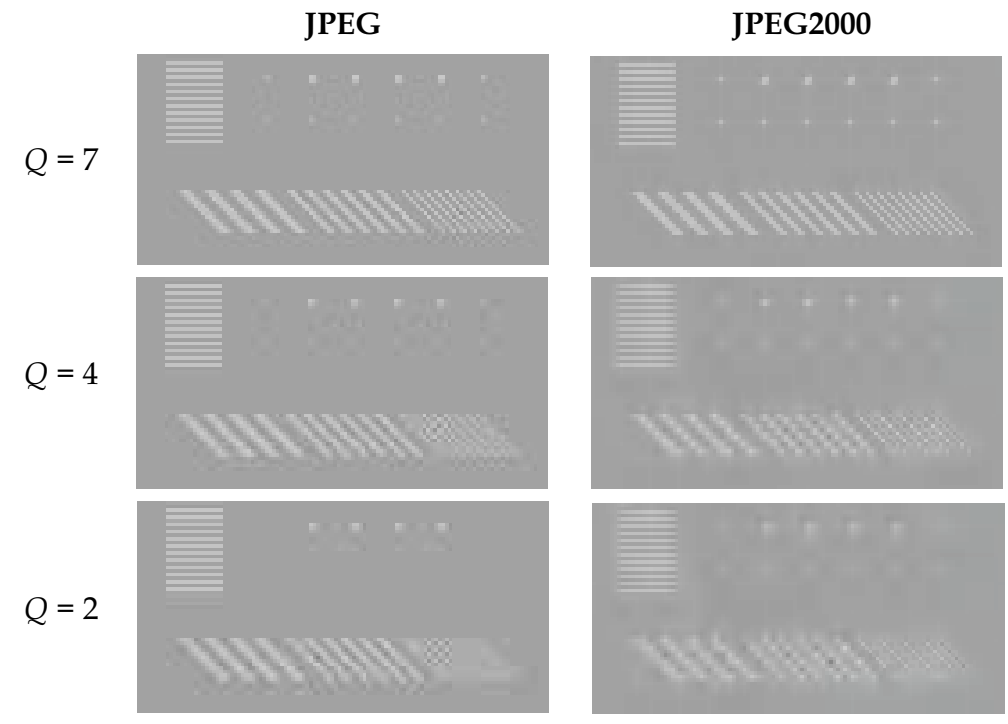

Figure 3. Fragments of the test image on brightness

The analysis of the received results allows making the following conclusion.

The JPEG and JPEG2000 codecs in ACDSee 8 provide higher compression factor of photo images in comparison with codecs of Adobe Photoshop CS at the established high quality reproduction of fine details.

\section{Quality analysis of MPEG-4 video images}

The experimental analysis of coding quality of dynamic images was carried out by the following technique (Sai, 2006).

At the first stage, test sequence of 12 frames $(360 \times 288)$ was transformed by means of Adobe Premiere 6.0 into a video clip without compression of video data with *.avi expansion.

At the second stage, the test video clip was compressed by the MPEG-4 Video compressor with the tuning dial ranging from 1 up to $100 \%$ for quality adjustment of video clips.

At the third stage, each frame of the compressed video clip was transformed into BMP format and passed into the program analyzer.

At the fourth stage, the quality of the decoded frames sequence was rated.

Figure 4 shows test sequence fragments on brightness (contrast is increased) for 1 and 6 frames, MPEG-4 Video algorithm for parameters Var $=90 \%$, Var $=70 \%$ and $\operatorname{Var}=50 \%$ having been executed.

Table 5 shows numerical results of quality rating estimation of the decoded sequence. Compression factors in relation to volume of the video data of the initial test sequence $(14,2$ $\mathrm{Mb})$ are also shown here.

Figure 5 shows fragments of the video clip frame with the real image after compression in MPEG-4 Video with high and low quality rating.

Visual comparison of images speaks to the fact that distortions of fine details (thin lines on the sweater) with low contrasts are practically imperceptible for vision at high quality rating. Low quality rating compression results in disappearance of low contrasts fine details from the image 

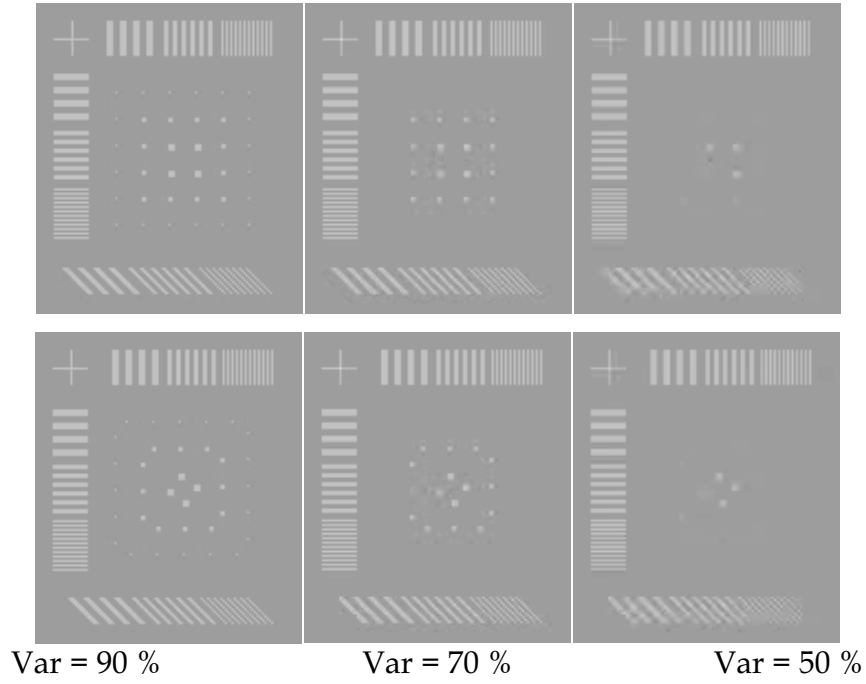

Figure 4 . Test sequence fragments on brightness

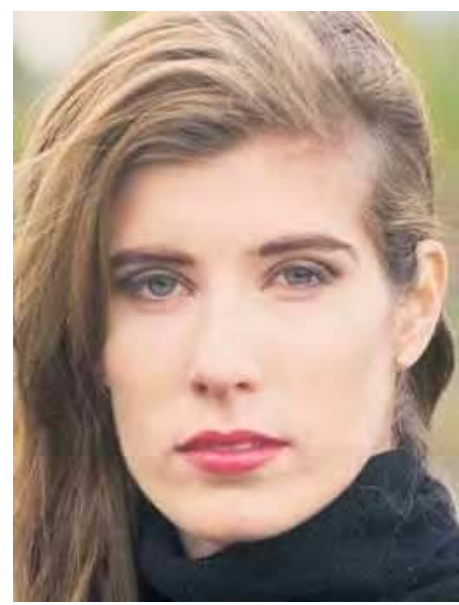

$Q=$ High

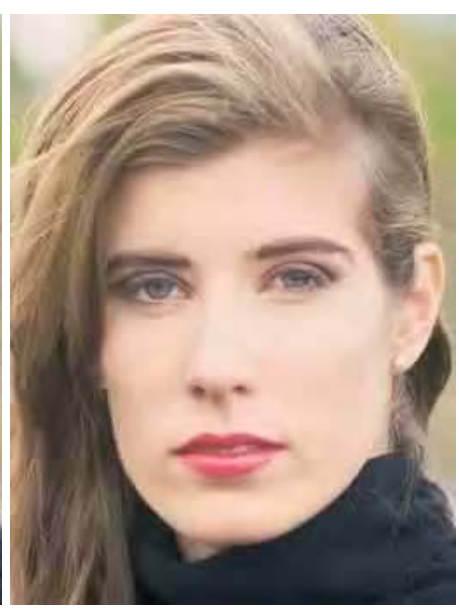

$Q=$ Low

Figure 5. Fragments of the video clip frame

\begin{tabular}{|c|c|c|c|}
\hline Var & $90 \%$ & $70 \%$ & $50 \%$ \\
\hline Rating & High & Medium & Low \\
\hline$Q$ & 6,8 & 4,3 & 2,7 \\
\hline$C f$ & 29,6 & 62,6 & 123,5 \\
\hline
\end{tabular}

Table 5. Quality rating MPEG-4

The received results lead to a conclusion that the adjustment scale should be established not less than $90 \%$ when using MPEG-4 Video with high reproduction quality of images fine details. 
Other types of MPEG-4 compressor are also investigated in this work.

In particular, it follows from the experimental results that the quality rating of the test sequence is 3,6 points for the DivX (Fast - Motion) compressor and 4,2 points for the DivX (Low - Motion). Thus, the use of these compressors results in average rating and does not allow receiving objectively high reproduction quality of video images fine details.

\section{Conclusion}

The developed objective methods of the definition analysis of images fine details practically prove to be effective and can be used for adjustment and optimization of codec parameters on high visual sharpness in various vision systems.

The main distinctive features of the developed methods should be noted in the summary.

1. The visual sharpness analysis is suggested to be carried out on the test image with low contrast, the initial contrast of fine details being set by a number of minimal color vision thresholds two - three times exceeding the average thresholds values.

2. Reduction in visual sharpness is suggested to be estimated by the normalized to vision thresholds, average value of test image fine details contrast deviation from the initial values of contrast in three-dimensional equal color space.

3. Image noise is suggested to be estimated by the root-mean-square values of color $W^{*}$, $U^{*}$ and $V^{*}$ coordinates deviations which are calculated by the quantity of minimal color vision thresholds.

To provide high quality reproduction of images fine details is the task of paramount importance at designing vision systems of various applications.

The author hopes that the methods offered in this work will help designers of vision systems to solve this task more effectively.

\section{References}

Glasman, K. (2004). MPEG-2 and Measurements. 625, No. 1, pp. 34-46, ISNN 0869-7914

Krivosheev, M.I. \& Kustarev, A.K. (1990). Color Measurements. Energoatom, Moscow, ISBN 5-283-00545-3.

Mac Adam, D.L. (1974). Uniform Color Scales. JOSA, Vol. 64, pp. 1691-1702.

Novakovsky, S.V. (1988). Color in Color TV. Radio and communication, Moscow, ISBN 5-25600090-X.

Pratt, W.K. (2001) Digital Image Processing. Wiley, ISBN 0471374075.

Sai, S.V. (2002). Definition Analysis of Color Static Images in Equal Contrast Space. Digital signals processing, No. 1., pp. 6-9, ISNN 1684-2634.

Sai, S.V. (2003). The Quality of Transmission and Reproduction of Fine Details in Color Television Images. Dalnauka, Vladivostok, ISBN 5-8044-0345-1.

Sai, S.V. (2006). Quality Analysis of MPEG-4 Video Images. Pattern Recognition and Image Analysis, Vol. 16, No. 1, pp. 50-51, ISNN 1054-6618.

Ventzel, E.S. \& Ovtharov, L.A. (2000). Probability Theory and its Engineering Application. Higher school, Moscow. ISBN 5-06-003830-0.

Wyszecki, G. (1975). Uniform Color Scales: CIE $1964 \mathrm{U}^{*} \mathrm{~V}^{*} \mathrm{~W}^{*}$ Conversion of OSA Committee Selection. JOSA, Vol. 65, pp. 456-460. 


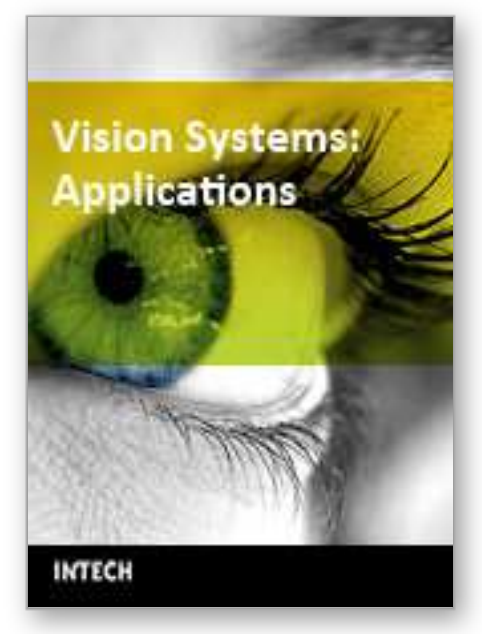

\author{
Vision Systems: Applications \\ Edited by Goro Obinata and Ashish Dutta
}

ISBN 978-3-902613-01-1

Hard cover, 608 pages

Publisher I-Tech Education and Publishing

Published online 01, June, 2007

Published in print edition June, 2007

Computer Vision is the most important key in developing autonomous navigation systems for interaction with the environment. It also leads us to marvel at the functioning of our own vision system. In this book we have collected the latest applications of vision research from around the world. It contains both the conventional research areas like mobile robot navigation and map building, and more recent applications such as, micro vision, etc. The fist seven chapters contain the newer applications of vision like micro vision, grasping using vision, behavior based perception, inspection of railways and humanitarian demining. The later chapters deal with applications of vision in mobile robot navigation, camera calibration, object detection in vision search, map building, etc.

\title{
How to reference
}

In order to correctly reference this scholarly work, feel free to copy and paste the following:

S.V. Sai (2007). Methods of the Definition Analysis of Fine Details of Images, Vision Systems: Applications, Goro Obinata and Ashish Dutta (Ed.), ISBN: 978-3-902613-01-1, InTech, Available from:

http://www.intechopen.com/books/vision_systems_applications/methods_of_the_definition_analysis_of_fine_d etails_of_images

\section{INTECH}

open science | open minds

\section{InTech Europe}

University Campus STeP Ri

Slavka Krautzeka 83/A

51000 Rijeka, Croatia

Phone: +385 (51) 770447

Fax: +385 (51) 686166

www.intechopen.com

\section{InTech China}

Unit 405, Office Block, Hotel Equatorial Shanghai

No.65, Yan An Road (West), Shanghai, 200040, China 中国上海市延安西路65号上海国际贵都大饭店办公楼 405 单元 Phone: $+86-21-62489820$

Fax: +86-21-62489821 
(C) 2007 The Author(s). Licensee IntechOpen. This chapter is distributed under the terms of the Creative Commons Attribution-NonCommercial-ShareAlike-3.0 License, which permits use, distribution and reproduction for non-commercial purposes, provided the original is properly cited and derivative works building on this content are distributed under the same license. 